\section{Pé Diabético: Avaliação da Evolução e Custo Hospitalar de Pacientes Internados no Conjunto Hospitalar de Sorocaba}

\section{RESUMO}

○é diabético é uma das principais complicações do diabetes mellitus (DM), sendo causa freqüente de internações hospitalares. Os custos destas internações e o ônus social constituem um grave problema de saúde. Com o objetivo de avaliar diabéticos portadores de lesões podais internados, estudamos 23 pacientes (14M, 9F; 39-80 anos de idade), no período de abril a novembro de 1999. Todos tinham DM tipo 2 e $48 \%$ deles foram internados com glicemia $<200 \mathrm{mg} / \mathrm{dL}$. As lesões estavam assim distribuídas (segundo classificação de Wagner): grau 1: 26\%; grau 2: 17\%; grau 3: 13\%; grau 4: 35\%; grau 5: 9\%. Evoluíram para amputação 65\% dos pacientes, sendo 30\% de coxa. $\bigcirc$ tempo mediano de internação foi de 14 dias. $\bigcirc$ custo mediano das internações foi de R\$1.004,59 e em 24\% delas foi superior a R\$2.000,00. Somado ao custo médio de próteses (R\$1.900,00), totaliza-se um ônus próximo a $\mathrm{R} \$ 3.000,00$. Na amostra, 9 pacientes estavam na faixa etária economicamente ativa. Os pacientes portadores de lesões nos pés são responsáveis por internações prolongadas e de custo elevado, o que não é compativel com o sistema público de saúde do nosso país. Assim, a prevenção é um dos pontos fundamentais para melhorar o prognóstico desta patologia. (Arq Bras Endocrinol Metab 2001;45/5:447-451)

Unitermos: Diabetes mellitus; Amputação; Lesões podais; Pé diabético; Gastos em saúde.

\begin{abstract}
Diabetic foot is one of the most important complications of diabetes mellitus (DM) and it is an ordinary cause of hospitalization. The costs of these hospitalizations and the social charge are serious health problems. To evaluate diabetic hospitalized patients with foot lesions, we studied 23 patients (14M, 9F; 39-80 years old), from April to November 1999. All had type 2 DM and $48 \%$ showed glucose levels $<200 \mathrm{mg} / \mathrm{dL}$ when admitted into the hospital. Foot lesions were categorized as follow (according to Wagner): grade 1: 26\%; grade 2: 17\%; grade 3: 13\%; grade 4: 35\%; grade 5: $9 \%$. Sixty-five percent of the patients evolved to amputations (30\% at thigh level). Median time of the hospitalization was 14 days. Median cost was R\$1,004 and in 24\% of the hospitalizations it was higher than $\mathrm{R} \$ 2,000$. If we aggregate the mean expenditure with prosthesis $(R \$ 1,900)$, the charges are around $R \$ 3,000$. Nine patients were at economically active age. Patients with foot lesions are responsible for long time hospitalizations with high costs, which is inadequate to the public health care system in our country. Consequently, prevention is absolutely significant to improve the prognosis of this pathology. (Arq Bras Endocrinol Metab 2001;45/5:447-451)
\end{abstract}

Keywords: Diabetes mellitus; Amputation; Diabetic foot; Health expenditures.

$\mathrm{O}$ DIABETES MELLITUS (DM) é um dos problemas mundiais de saúde mais importantes da atualidade, por ser uma doença com elevada morbidade e mortalidade (1-3). Uma de suas complicações mais fre-

\section{artigo original}

\author{
Mauro H.S.A. Milman \\ Cristina B.M. Leme \\ Danilo T. Bovelli \\ Fábio R. Kater \\ Elizabeth C.D.C. Baccili \\ Rita C.M. Rocha \\ Maria-Helena Senger
} Disciplina de Endocrinologia,
Faculdade de Ciências Médicas de Sorocaba-
CCMB/PUC-SP. 
qüentes é o pé diabético, caracterizado pela presença de lesões nos pés em decorrência das alterações vasculares e/ou neurológicas peculiares do DM (3-7). Trata-se de uma complicação crônica que ocorre em média após 10 anos de evolução do DM e é a causa mais comum de amputações não traumáticas $(1,4,8$ 12). Por isso, sugere-se que uma intervenção intensiva possa prevenir o aparecimento ou atenuar a evolução do pé diabético $(13,14)$.

Fatores como idade, tipo e tempo de diagnóstico do DM, controle metabólico, tabagismo, alcoolismo, obesidade, hipertensão arterial e falta de bons hábitos higiênicos no cuidado com os pés são importantes quanto ao risco dessa complicação. Tais fatores favorecem a formação de úlcera, infecção e gangrena, podendo culminar em amputação $(1,7,10,13,15,16)$.

O pé diabético é responsável por parcela significativa das internações de pacientes diabéticos, constituindo-se também na maior causa de hospitalizações prolongadas nestes pacientes $(1,3)$. No Brasil, o DM também é causa importante de amputações de membros inferiores, sendo um considerável fator de incapacidade, invalidez, aposentadoria precoce e mortes evitáveis $(1,17)$. Além destes graves problemas, devese levar em consideração os gastos e as internações prolongadas que causam grande prejuízo ao sistema público de saúde $(7,10)$.

Os objetivos deste trabalho foram a identificação dos pacientes com pé diabético internados nas enfermarias do Conjunto Hospitalar de Sorocaba e a avaliação da sua evolução e do custo hospitalar de suas internações.

\section{MATERIAL E MÉTODOS}

Realizou-se um estudo prospectivo de pacientes apresentando lesões de membros inferiores relacionadas ao DM e internados nas enfermarias do Conjunto Hospitalar de Sorocaba (CHS) no período de abril a novembro de 1999. O CHS é constituído por dois hospitais-escola da Secretaria de Estado da Saúde conveniados à Faculdade de Ciências Médicas de Sorocaba (PUC-SP). Os pacientes são atendidos através do Sistema Único de Saúde (SUS).

Cada paciente respondeu a um questionário onde constavam dados sobre o tempo de diagnóstico do DM, presença de outras doenças, ocorrência de complicações, forma de acompanhamento e tratamento da doença até a internação atual.

Além do exame físico geral, foi realizada uma avaliação dos membros inferiores e as lesões foram ordenadas, no momento da internação, de acordo com a classificação de Wagner (tabela 1) $(10,13)$.
Tabela 1. Classificação de Wagner para as lesões do pé diabético $(10,13)$.

\begin{tabular}{ll}
\hline Grau & Características \\
\hline 0 & $\begin{array}{l}\text { Nenhuma úlcera evidente, com calosidades gros- } \\
\text { sas e cabeças metatársicas proeminentes, dedos }\end{array}$ \\
& em garra ou outras anormalidades ósseas \\
1 & Úlcera superficial sem infecção evidente \\
3 & Úlcera profunda sem envolvimento ósseo \\
& Úlcera profunda com formação de abscesso ou \\
4 & envolvimento ósseo \\
5 & Gangrena localizada \\
\hline
\end{tabular}

Em todos os pacientes também foi realizada a glicemia por ocasião da internação, dosada pelo método enzimático. Além disto, avaliou-se a evolução das lesões e o custo hospitalar das internações, considerando-se os valores cobrados do SUS no período da pesquisa, referentes à soma de diárias hospitalares, medicações, serviços profissionais e exames subsidiários. Foram consideradas internações prolongadas aquelas com duração superior à permitida pela Tabela de Procedimentos por Grupo do SUS, vigente durante a pesquisa, e que determinava o período de 8 dias como o tempo máximo de internação nos casos de pé diabético e, excepcionalmente, 14 dias para os submetidos à amputação de coxa ou perna ou à bypass vascular fềmuro-poplíteo.

A presente pesquisa foi aprovada pelo Comitê de Ética em Pesquisa local e os pacientes, após serem esclarecidos, forneceram consentimento assinado.

Os resultados foram submetidos à análise estatística pelos seguintes testes não-paramétricos: Qui Quadrado, Mann-Whitney e Kruskal-Wallis. As diferenças foram consideradas significantes quando $\mathrm{p}<0,05$.

\section{RESULTADOS}

Vinte e três pacientes participaram do estudo, sendo 14 (61\%) do sexo masculino e 9 (39\%) do feminino. A idade variou entre 39 e 80 anos (mediana: 69 anos). Todos os pacientes eram portadores de DM tipo 2. Onze doentes (48\%) eram tabagistas e $6(26 \%)$, etilistas.

Quatro pacientes (17\%) desconheciam ser diabéticos até a internação. Dentre os outros, $6(26 \%)$ tinham menos de 10 anos de diagnóstico do DM; 6 (26\%) entre 10 e 15 anos e 7 (30\%), mais de 15 anos.

A hipertensão arterial foi referida por 14 pacientes $(61 \%)$ e as dislipemias por 5 (22\%). Quando interrogados, as queixas apresentadas sugeriram a ocorrência de neuropatia autonômica em 18 pacientes $(78 \%)$, neuropatia periférica em $17(74 \%)$, arteriopatia em $16(70 \%)$, retinopatia em 5 (22\%) e nefropatia em 4 (17\%). 


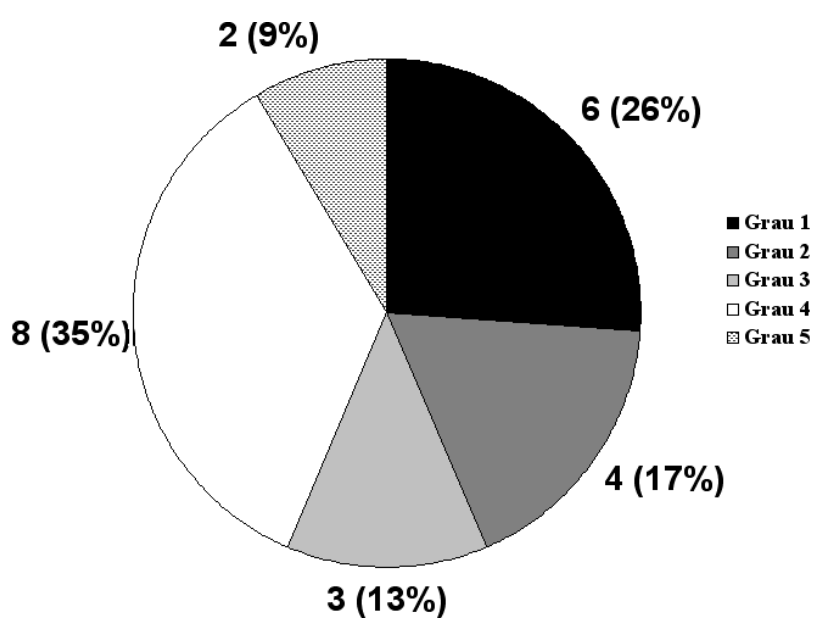

Gráfico 1. Distribuição dos pacientes internados com pé diabético no CHS, de acordo com a classificação de Wagner.

Todos os pacientes sabidamente diabéticos eram tratados com medidas higieno-dietéticas e medicamentos: $13(56 \%)$ com hipoglicemiantes orais $(26 \%$, clorpropamida; $17 \%$, glibenclamida; $9 \%$, glimepirida; $4 \%$, metformina) e $6(26 \%)$ com insulina. Aos esquemas prescritos, 11 pacientes $(48 \%)$ diziam-se aderentes.

O local de acompanhamento desses pacientes foi variável: 9 (39\%) faziam acompanhamento em postos de saúde, 4 (17\%) em ambulatórios de endocrinologia e/ou cirurgia vascular e 5 (22\%) em outros serviços. Apenas um (4\%) referiu não procurar atendimento médico rotineiramente.

Quanto ao estadiamento das lesões, nenhum paciente apresentou lesão grau 0 quando internado. A distribuição dos pacientes segundo a classificação de Wagner está apresentada no gráfico 1 . A maior parte dos pacientes $(n=8 ; 35 \%)$ apresentava lesões grau 4 . As lesões eram recidivantes em 39\% dos casos.

Apenas 11 pacientes $(48 \%)$ apresentavam glicemia inferior a $200 \mathrm{mg} / \mathrm{dL}$, sendo que 3 (13\%) pacientes tinham valores maiores que $300 \mathrm{mg} / \mathrm{dL}$. Nenhum paciente se encontrava em descompensação aguda do DM.

Cinco pacientes $(22 \%)$ foram tratados clinicamente, tendo 1 ( $4 \%$ ) evoluído a óbito no $8^{\circ}$ dia de internação por infarto agudo do miocárdio. As intervenções cirúrgicas realizadas em 18 pacientes $(78 \%)$ estão apresentadas no gráfico 2 . Um deles foi submetido a dois procedimentos cirúrgicos: construção de enxerto vascular (fêmuro-poplíteo) e amputação de antepé. Do total de 23 pacientes, 15 (65\%) evoluíram para amputações.

O tempo de internação variou de 3 a 61 dias (mediana: 14 dias). Dos 13 pacientes (56\%) que deveri-

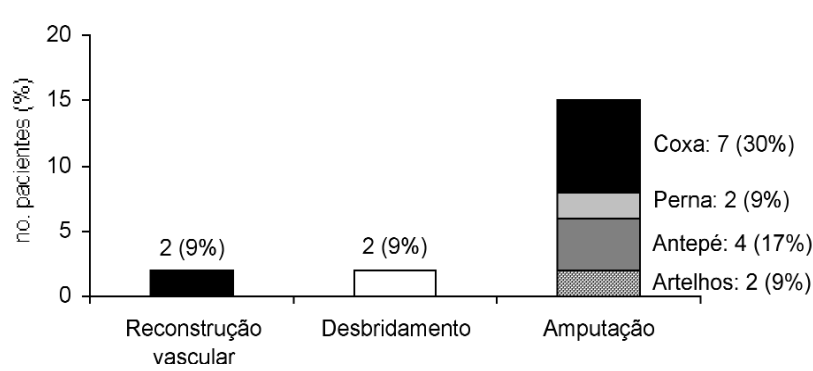

Gráfico 2. Intervenções cirúrgicas realizadas em 18 pacientes internados com pé diabético no CHS e nível das amputações realizadas.

am permanecer no hospital por, no máximo, 8 dias, 6 (26\%) permaneceram por um tempo maior (11 a 30 dias). Dos 10 (43\%) submetidos a amputações de perna ou coxa ou à reconstrução vascular, 7 (30\%) ficaram internados além dos 14 dias (17 a 61) máximos determinados pelo SUS. Assim, 13 internações (56\%) estudadas prolongaram-se além do estabelecido pelo SUS.

Considerando-se 21 pacientes, o gasto individual variou de $\mathrm{R} \$ 211,16$ a $\mathrm{R} \$ 7.164,05$ (mediana: R\$1.004,59). Dez internações (47\%) tiveram um custo inferior a $\mathrm{R} \$ 1.000,00$; seis $(29 \%)$ entre $\mathrm{R} \$ 1.000,00$ e $R \$ 2.000,00$ e cinco (24\%) superior a $\mathrm{R} \$ 2.000,00$. Não foi possível obter o custo hospitalar de duas internações.

A análise estatística não mostrou associação das seguintes variáveis quando estudadas aos pares: idade do paciente, tempo de diagnóstico do DM, nível glicêmico, severidade ou recorrência da lesão, nível da amputação, aderência ao tratamento, tempo de internação e custo hospitalar $(\mathrm{p}>0,05)$.

\section{DISCUSSÃO}

Este estudo mostrou que o pé diabético é uma patologia grave em nosso meio, culminando com uma alta taxa de amputações, internações prolongadas e altos custos hospitalares. O grupo de pacientes apresentado representa a maioria dos diabéticos propensos a desenvolver lesões em membros inferiores: aqueles com DM tipo 2, idosos e com mais de 10 anos de diagnóstico da doença $(10-12,15,18)$.

O tempo de diagnóstico do DM foi próximo ao apresentado em outros estudos da literatura, apesar da variabilidade do período de latência clínica desta doença, durante o qual já podem estar ocorrendo o desenvolvimento de complicações crônicas como o pé diabético $(12,15)$. O percentual de pacientes que desconhecia ser portador de DM (17\%) foi mais elevado que os $10 \%$ relatados em um levantamento re- 
trospectivo brasileiro realizado em 70 pacientes (15). Isto indica que o diagnóstico do DM tipo 2 ainda vem sendo feito quando já são evidentes algumas complicações crônicas, como o pé diabético, em uma parcela considerável de casos.

Os sintomas de outras complicações do DM foram freqüentemente apontados pelos pacientes estudados. Estes dados representam apenas as queixas dos pacientes, pois o estudo de outras possíveis complicações associadas não foi objeto deste levantamento. Apesar disso, eles sugerem o que já foi demonstrado na literatura: a alta prevalência de complicações crônicas do DM associadas ao pé diabético, como a arteriopatia e a neuropatia $(4-7,10)$.

Também chama a atenção o fato de que mais da metade dos pacientes referiram falta de aderência à terapêutica anteriormente indicada nos locais onde faziam acompanhamento, comparável ao descrito em estudo realizado na cidade de São Paulo (1), que apontou uma taxa de não-aderência de $62 \%$. Isto sem dúvida tende a contribuir para o mau controle glicêmico e desenvolvimento de complicações, apesar de que em nossa pequena amostra não foi encontrada significância estatística.

A gravidade das lesões foi heterogênea, havendo desde úlceras superficiais até gangrenas extensas. Lesões grau 4 foram as mais freqüentes, semelhante ao relatado em outros estudos $(10,12,15)$. A evolução das lesões mostrou a necessidade de intervenções mais amplas, culminando em uma elevada taxa de amputações $(65 \%)$, predominando as de coxa, conforme resultados já descritos $(1,2,10,12)$.

A mediana do tempo de internação (14 dias) na amostragem pode ser considerada elevada, principalmente pela grande proporção de internações prolongadas $(56 \%)$. Contudo, este período foi significativamente inferior aos relatados em outros levantamentos: 23 a 38 dias $(2,9,11,12,15)$. Esta diferença pode se dever a disparidades em termos de gravidade dos casos, tempo de acompanhamento e número de pacientes estudados.

Considerando-se apenas os 9 pacientes (39\%) que se encontravam na faixa etária economicamente ativa, houve uma perda total de 126 dias de trabalho (variando de 5 a 29 dias), devido apenas às internações hospitalares. Além disso, 5 destes pacientes sofreram amputações de coxa ou antepé, o que acarretará comprometimento da capacidade de trabalho.

Cerca de $25 \%$ das internações tiveram um custo superior a $\mathrm{R} \$ 2.000,00$. Ressalte-se que este valor foi auferido através da Tabela de procedimentos por Grupo do SUS, que por exemplo determina o valor total de $\mathrm{R} \$ 498,84$ para a amputação de perna (incluindo serviços hospitalares, profissionais, medicamentos e exames laboratoriais para a permanência de 7 dias) e de $\mathrm{R} \$ 153,28$ para a internação por 4 dias de um paciente com DM.

No grupo de diabéticos que sofreram amputação de coxa ou perna, haveria ainda um gasto adicional de cerca de $\mathrm{R} \$ 1.900,00$ com próteses por paciente, que somados ao custo mediano de internação $(\mathrm{R} \$ 1.004,59)$, totalizaria uma despesa próxima a $\mathrm{R} \$ 3.000,00$. Deve-se lembrar que estes doentes necessitam também de inúmeras sessões de fisioterapia, consultas médicas (endocrinologista e fisiatra) e, de acordo com o caso, cirurgião vascular e psicólogo $(15,19)$. Um levantamento realizado entre os serviços privados americanos estimou um custo total de US $\$ 33.444,00$ por paciente submetido à amputação de membro inferior, incluindo-se os gastos com tratamento e recuperação $(9)$.

A falta de correlação estatística entre os parâmetros estudados pode ser conseqüente à pequena amostragem estudada, pois já foi demonstrada a associação entre a não-aderência e a recorrência de lesões (9).

Assim, este estudo demonstrou que os pacientes portadores de pé diabético necessitam de internações prolongadas e de custo elevado, quando analisadas segundo a tabela SUS, nem sempre compatíveis com o sistema público de saúde de nosso país. Estes doentes também têm a qualidade de vida comprometida, como conseqüência não só das internações e faltas ao trabalho como também da deficiência física gerada pelas amputações. Portanto, a prevenção adequada desta complicação do DM torna-se obrigatória, pelo diagnóstico mais precoce do $\mathrm{DM}$, rigoroso controle metabólico e orientações para os cuidados com os pés, cabendo ao médico identificar os pacientes mais propensos ao seu desenvolvimento (20).

\section{REFERÊNCIAS}

1. Gamba MA. Amputações por diabetes mellitus: uma prática prevenível. Acta Paul Enf 1998;11:92-100.

2. Tagle M, Francisco V, Gómez F, Anchudia O. Morbi-mortalidad y tratamiento en pacientes hospitalizados por pie diabético. Educ Med Contin 1995;48:14-9.

3. Zervos M. Treatment of polymicrobial intra-abdominal, pelvic and diabetic foot infections. Comp Ther 1998;24:295-301.

4. Foster A. Diabetes care: getting your patient on sure footing. Nurs Times 1999:95:51-2.

5. Morgan CLI, Currie CJ, Scott NCH, Smithers M, Butler CC, Peters JR. The prevalence of multiple diabetes-related complications. Diabet Med 2000; 17:146-51. 
6. Tomas MB, Patel M, Marwin SE, Palestro CJ. The diabetic foot. Br J Radiol 2000;73:443-50.

7. Zangaro GA, Hull MM. Diabetic neuropathy: pathophisiology and prevention of foot ulcers. Clin Nurs Spec 1999: 13:57-65

8. Pinzur MS, Shields NN, Goelitz B, Slovenkai M, Kaye R, Ross SDK, et al. American orthopaedic foot and ankle society shoe survey of diabetic patients. Foot Ankle Int 1999:20:703-7.

9. Aguila MAD, Reiber GE, Koepsell TD. How does provider and patient awareness of high-risk status for lowerextremity amputation influence foot-care practice? Diabetes Care 1994;17:1050-4.

10. Alcántara W, Flores R, Garmendia F. Prevalencia y riesgo de amputación en pacientes con pie diabético. An Fac Med (Perú) 1999;60:159-64.

11. Olea LS, Cabral C, Campero GC, Olaya L, Estrada J, Fuentes A. Pie diabético: criterios de prevención, diagnostico y tratamiento desde el punto de vista ortopedico. Rev Med Tucumán 1994;1:297-312.

12. Vilca F, Angel M. Estudio de pacientes amputados por pie diabético en el Servicio de Ortopedia y Tramatología el Hospital Regional Honorio Delgado entre 1985-1995. Arequipa 1997;48 p.

13. Levin ME. Classification of diabetic foot wounds. Diabetes Care 1998;21:681-2.

14. Mayfield JA, Strand T, Toya AR. A call for specific codes for diabetes foot and eye care. Diabetes Care $1995 ; 18: 418-21$.
15. Jorge BH, Borges MF, Brito VN, Santos TGM, Thirone ACP. Análise clínica e evolução de 70 casos de lesões podais infectados em pacientes diabéticos. Arq Bras Endocrinol Metab 1999:43:366-72.

16. LoGerfo FW, Gibbons GW. Vascular disease of the lower extremities in diabetes mellitus. Endocrinol Metab Clin North Amer 1996;25:439-45.

17. Spichler D, Spichler ERS, Forti AC, Lessa I, Franco LJ Amputações de membros inferiores - Rio de Janeiro, RJ, 1994-1996. Arq Bras Endocrinol Metab 1999;43:s262.

18. Thomaz JB, Herdy CDC, Brancaglion SB, Oliveira JCP, Thomaz YCM, Lima MV, et al. Pé diabético. Ars Curandi 1996; abril:51-104.

19. Wagner FW. The diabetic foot: a personal experience of 50 years. Foot Ankle Int 1999:20:684-6.

20. American Diabetes Association: Consensus Development Conference on Diabetic Foot Wound Care (consensus statement). Diabetes Care 1999;1354-60.

\section{Endereço para correspondência:}

Maria Helena Senger

Av. São Paulo 2918

18.013-004 Sorocaba, SP

Fax: (15) 227-2565

e.mail: mhsenger@dglnet.com.br 